\title{
Numerical Solution of the Blasius Viscous Flow Problem by Quartic B-Spline Method
}

\author{
Hossein Aminikhah and Somayyeh Kazemi \\ Department of Applied Mathematics, Faculty of Mathematical Sciences, University of Guilan, P.O. Box 1914, Rasht 41938, Iran \\ Correspondence should be addressed to Hossein Aminikhah; hossein.aminikhah@gmail.com
}

Received 1 March 2016; Accepted 28 March 2016

Academic Editor: Josè A. Tenereiro Machado

Copyright (c) 2016 H. Aminikhah and S. Kazemi. This is an open access article distributed under the Creative Commons Attribution License, which permits unrestricted use, distribution, and reproduction in any medium, provided the original work is properly cited.

\begin{abstract}
A numerical method is proposed to study the laminar boundary layer about a flat plate in a uniform stream of fluid. The presented method is based on the quartic B-spline approximations with minimizing the error $L_{2}$-norm. Theoretical considerations are discussed. The computed results are compared with some numerical results to show the efficiency of the proposed approach.
\end{abstract}

\section{Introduction}

One of the well-known equations arising in fluid mechanics and boundary layer approach is Blasius differential equation. The classical Blasius [1] equation is a third-order nonlinear two-point boundary value problem, which describes twodimensional incompressible laminar flow over a semi-infinite flat plate at high Reynolds number,

$$
2 f^{\prime \prime \prime}(x)+f(x) f^{\prime \prime}(x)=0, \quad x \geq 0,
$$

with boundary conditions

$$
\begin{array}{r}
f(0)=0, \\
f^{\prime}(0)=0, \\
\lim _{x \rightarrow \infty} f^{\prime}(x)=1,
\end{array}
$$

where the prime denotes the derivatives with respect to $x$. In addition to the unknown function $f$, the solution of (1) and (2) is characterized by the value of $\alpha=f^{\prime \prime}(0)$. Blasius [1] in 1908 found the exact solution of boundary layer equation over a flat plate. A highly accurate numerical solution of Blasius equation has been provided by Howarth [2], who obtained the initial slope $\alpha=f_{\text {ex }}^{\prime \prime}(0)=0.332057$. Liu and Chang [3] have developed a new numerical technique; they have transformed the governing equation into a nonlinear secondorder boundary value problem by a new transformation technique, and then they have solved it by the Lie group shooting method. He $[4,5]$ gave a solution in a family of power series with parameter $p$ by means of the perturbation method for solving this equation. Bender et al. [6] proposed a simple approach using $\delta$-expansion to obtain accurate totally analytical solution of viscous fluid flow over a flat plate. Aminikhah [7] used LTNHPM to obtain an analytical approximation to the solution of nonlinear Blasius viscous flow equation. Recently, the fixed point method (FPM) [8], which is based on the fixed point concept in functional analysis, is adopted to acquire the explicit approximate analytical solution to the nonlinear differential equation. Finally, efforts $[9,10]$ have been made to obtain the solution at the surface boundary and changed the problem from a boundary value differential equation into an initial value one. The solution in the entire domain, however, still requires computation.

In this paper, the quartic B-spline approximations are employed to construct the numerical solution for solving the Blasius equation. The unknowns are obtained with using optimization. The proposed method is applied to the problem and the computed results are compared with those of Howarth's method to demonstrate its efficiency.

\section{Description of the Method}

Let there be a uniform partition of an interval $[0, L]$ as follows: $0=x_{0}<x_{1}<\cdots<x_{N-1}<x_{N}=L$, where $h=x_{j+1}-x_{j}$, $j=0,1, \ldots, N-1$. The quartic B-splines are defined upon 
an increasing set of $N+1$ knots over the problem domain plus 8 additional knots outside the problem domain; the 8 additional knots are positioned as

$$
\begin{aligned}
& x_{-4}<x_{-3}<x_{-2}<x_{-1}<x_{0}, \\
& x_{N}<x_{N+1}<x_{N+2}<x_{N+3}<x_{N+4} .
\end{aligned}
$$

The quartic B-splines $B_{j}(x), j=-2,-1, \ldots, N+1$, at the knots $x_{j}$ are defined as [12]

$$
B_{j}(x)=\frac{1}{24 h^{4}} \begin{cases}\left(x-x_{j-2}\right)^{4}, & x_{j-2} \leq x<x_{j-1}, \\ h^{4}+4 h^{3}\left(x-x_{j-1}\right)+6 h^{2}\left(x-x_{j-1}\right)^{2}+4 h\left(x-x_{j-1}\right)^{3}-4\left(x-x_{j-1}\right)^{4}, & x_{j-1} \leq x<x_{j}, \\ 11 h^{4}+12 h^{3}\left(x-x_{j}\right)-6 h^{2}\left(x-x_{j}\right)^{2}-12 h\left(x-x_{j}\right)^{3}+6\left(x-x_{j}\right)^{4}, & x_{j} \leq x<x_{j+1}, \\ h^{4}+4 h^{3}\left(x_{j+2}-x\right)+6 h^{2}\left(x_{j+2}-x\right)^{2}+4 h\left(x_{j+2}-x\right)^{3}-4\left(x_{j+2}-x\right)^{4}, & x_{j+1} \leq x<x_{j+2}, \\ \left(x_{j+3}-x\right)^{4}, & x_{j+2} \leq x<x_{j+3}, \\ 0, & \text { otherwise. }\end{cases}
$$

And the set $\left\{B_{-2}, B_{-1}, \ldots, B_{N+1}\right\}$ of quartic B-splines forms a basis over the region $0 \leq x \leq L$.

Let $S(x)$ be the quartic B-spline function at the nodal points. Then approximate solution of (1) can be written as [13]

$$
S(x)=\sum_{j=-2}^{N+1} C_{j} B_{j}(x),
$$

where $B_{j}(x)$ are the quartic B-spline functions and $C_{j}$ are the unknown coefficients. Each B-spline covers the five elements so that an element is covered by five B-splines. The values of $B_{j}(x)$ and its derivatives are tabulated in Table 1.

Then from (5) we have

$$
\begin{aligned}
S^{\prime}(x) & =\sum_{j=-2}^{N+1} C_{j} B_{j}^{\prime}(x), \\
S^{\prime \prime}(x) & =\sum_{j=-2}^{N+1} C_{j} B_{j}^{\prime \prime}(x), \\
S^{\prime \prime \prime}(x) & =\sum_{j=-2}^{N+1} C_{j} B_{j}^{\prime \prime \prime}(x) .
\end{aligned}
$$

Using Table 1 in (5)-(6), we obtained

$$
\begin{aligned}
S\left(x_{j}\right)= & \left(\frac{1}{24}\right) C_{j-2}+\left(\frac{11}{24}\right) C_{j-1}+\left(\frac{11}{24}\right) C_{j} \\
& +\left(\frac{1}{24}\right) C_{j+1}, \\
S^{\prime}\left(x_{j}\right)= & \left(\frac{-1}{6 h}\right) C_{j-2}+\left(\frac{-1}{2 h}\right) C_{j-1}+\left(\frac{1}{2 h}\right) C_{j} \\
& +\left(\frac{1}{6 h}\right) C_{j+1},
\end{aligned}
$$

$$
\begin{aligned}
S^{\prime \prime}\left(x_{j}\right)= & \left(\frac{1}{2 h^{2}}\right) C_{j-2}+\left(\frac{-1}{2 h^{2}}\right) C_{j-1}+\left(\frac{-1}{2 h^{2}}\right) C_{j} \\
& +\left(\frac{1}{2 h^{2}}\right) C_{j+1}, \\
S^{\prime \prime \prime}\left(x_{j}\right)= & \left(\frac{-1}{h^{3}}\right) C_{j-2}+\left(\frac{3}{h^{3}}\right) C_{j-1}+\left(\frac{-3}{h^{3}}\right) C_{j} \\
& +\left(\frac{1}{h^{3}}\right) C_{j+1},
\end{aligned}
$$

where $j=0,1, \ldots, N$. Substituting (7) into (1) and (2) and by assuming initial slope $f^{\prime \prime}(0)=0.332057$, similar to Howarth, we have

$$
\begin{aligned}
S\left(x_{0}\right) & =0, \\
S^{\prime}\left(x_{0}\right) & =0, \\
S^{\prime \prime}\left(x_{0}\right) & =0.332057, \\
S^{\prime \prime \prime}\left(x_{j}\right)+\frac{1}{2} S\left(x_{j}\right) S^{\prime \prime}\left(x_{j}\right) & =0, \quad j=0,1, \ldots, N .
\end{aligned}
$$

Then, we get a system of $(n+4)$ nonlinear equations in the $(n+4)$ unknowns $C_{-2}, C_{-1}, \ldots, C_{N+1}$.

In order to solve system (8), we direct attention to that $S(x)$ and its derivatives satisfied in (1) and (2) and initial slope $f^{\prime \prime}(0)=0.332057$ approximately, and then we have

$$
\begin{aligned}
S\left(x_{0}\right) & \approx 0, \\
S^{\prime}\left(x_{0}\right) & \approx 0, \\
S^{\prime \prime}\left(x_{0}\right) & \approx 0.332057, \\
S^{\prime \prime \prime}\left(x_{j}\right)+\frac{1}{2} S\left(x_{j}\right) S^{\prime \prime}\left(x_{j}\right) & \approx 0, \quad j=0,1, \ldots, N .
\end{aligned}
$$


TABLE 1: Coefficient of quartic B-splines and its derivatives at knots $x_{j}$.

\begin{tabular}{lcccccc}
\hline$x$ & $x_{j-2}$ & $x_{j-1}$ & $x_{j}$ & $x_{j+1}$ & $x_{j+2}$ & $x_{j+3}$ \\
\hline$B_{j}(x)$ & 0 & $\frac{1}{24}$ & $\frac{11}{24}$ & $\frac{11}{24}$ & $\frac{1}{24}$ & 0 \\
$B_{j}^{\prime}(x)$ & 0 & $\frac{1}{6 h}$ & $\frac{1}{2 h}$ & $-\frac{1}{2 h}$ & $-\frac{1}{6 h}$ & 0 \\
$B_{j}^{\prime \prime}(x)$ & 0 & $\frac{1}{2 h^{2}}$ & $-\frac{1}{2 h^{2}}$ & $-\frac{1}{2 h^{2}}$ & $\frac{1}{2 h^{2}}$ & 0 \\
$B_{j}^{\prime \prime \prime}(x)$ & 0 & $\frac{1}{h^{3}}$ & $-\frac{3}{h^{3}}$ & $\frac{3}{h^{3}}$ & $-\frac{1}{h^{3}}$ & 0 \\
\hline
\end{tabular}

Therefore, the error vector $E$ of the approximation can be written as

$$
\begin{aligned}
E_{1} & =S\left(x_{0}\right), \\
E_{2} & =S^{\prime}\left(x_{0}\right), \\
E_{3} & =S^{\prime \prime}\left(x_{0}\right)-0.332057, \\
E_{j+4} & =S^{\prime \prime \prime}\left(x_{j}\right)+\frac{1}{2} S\left(x_{j}\right) S^{\prime \prime}\left(x_{j}\right), \quad j=0,1, \ldots, N .
\end{aligned}
$$

Now, we wish to minimize the error norm, the $L_{2}$-norm, such that

$$
L_{2}=\sqrt{\sum_{j=1}^{N+4} E_{j}^{2}}=0 .
$$

By solving (11), we can get the values of $C_{j}$ for $j=-2,-1, \ldots, N+1$. Now, with substituting the values $C_{-2}, C_{-1}, C_{0}, \ldots, C_{N+1}$ into (7), the approximate value of $S(x)$ and its derivatives will be ensured.

For calculating the truncation error of this method, from (7), the following relationships can be obtained [14]:

$$
\begin{aligned}
h & \left.S^{\prime}\left(x_{j-2}\right)+11 S^{\prime}\left(x_{j-1}\right)+11 S^{\prime}\left(x_{j}\right)+S^{\prime}\left(x_{j+1}\right)\right] \\
= & 4\left[S\left(x_{j+1}\right)+3 S\left(x_{j}\right)-3 S\left(x_{j-1}\right)-S\left(x_{j-2}\right)\right], \\
h^{2} S^{\prime \prime}\left(x_{j}\right) & \\
= & 2\left[S\left(x_{j+1}\right)-2 S\left(x_{j}\right)+S\left(x_{j-1}\right)\right] \\
& -\frac{h}{2}\left[S^{\prime}\left(x_{j+1}\right)-S^{\prime}\left(x_{j-1}\right)\right], \\
h^{3} S^{\prime \prime \prime} & \left(x_{j}\right) \\
= & 12\left[S\left(x_{j+1}\right)-S\left(x_{j-1}\right)\right] \\
& -3 h\left[S^{\prime}\left(x_{j+1}\right)+6 S^{\prime}\left(x_{j}\right)+S^{\prime}\left(x_{j-1}\right)\right],
\end{aligned}
$$

and, then, we have

$$
\begin{aligned}
S^{\prime}\left(x_{j}\right) & =f^{\prime}\left(x_{j}\right)+\frac{h^{4}}{720} f^{(5)}\left(x_{j}\right)+O\left(h^{6}\right), \\
S^{\prime \prime}\left(x_{j}\right) & =f^{\prime \prime}\left(x_{j}\right)-\frac{h^{4}}{240} f^{(6)}\left(x_{j}\right)+O\left(h^{6}\right), \\
S^{\prime \prime \prime}\left(x_{j}\right)= & f^{\prime \prime \prime}\left(x_{j}\right)-\frac{h^{2}}{12} f^{(5)}\left(x_{j}\right)+\frac{h^{4}}{240} f^{(7)}\left(x_{j}\right) \\
& +O\left(h^{6}\right) .
\end{aligned}
$$

Therefore, truncation error is defined as follows:

$$
\begin{aligned}
& e\left(x_{j}\right)=\left[S^{\prime \prime \prime}\left(x_{j}\right)+\frac{1}{2} S\left(x_{j}\right) S^{\prime \prime}\left(x_{j}\right)\right]-\left[f^{\prime \prime \prime}\left(x_{j}\right)\right. \\
& \left.+\frac{1}{2} f\left(x_{j}\right) f^{\prime \prime}\left(x_{j}\right)\right]=\left[S^{\prime \prime \prime}\left(x_{j}\right)-f^{\prime \prime \prime}\left(x_{j}\right)\right] \\
& +\frac{1}{2}\left[S\left(x_{j}\right) S^{\prime \prime}\left(x_{j}\right)-f\left(x_{j}\right) f^{\prime \prime}\left(x_{j}\right)\right] \\
& \quad=\left[-\frac{h^{2}}{12} f^{(5)}\left(x_{j}\right)+\frac{h^{4}}{240} f^{(7)}\left(x_{j}\right)+O\left(h^{6}\right)\right] \\
& +\left[-\frac{h^{4}}{480} f\left(x_{j}\right) f^{(6)}\left(x_{j}\right)+\frac{h^{6}}{12096} f\left(x_{j}\right) f^{(8)}\left(x_{j}\right)\right. \\
& \left.+O\left(h^{8}\right)\right], \quad j=1,2, \ldots, N .
\end{aligned}
$$

Thus, for $j=1,2, \ldots, N$, we have

$$
\begin{aligned}
e\left(x_{j}\right)= & {\left[-\frac{f^{(5)}\left(x_{j}\right)}{12}\right] h^{2} } \\
& +\left[\frac{2 f^{(7)}\left(x_{j}\right)-f\left(x_{j}\right) f^{(6)}\left(x_{j}\right)}{480}\right] h^{4} \\
& +O\left(h^{6}\right), \quad j=1,2, \ldots, N,
\end{aligned}
$$

and, for $x=0$, we have

$$
\begin{aligned}
e\left(x_{0}\right)= & {\left[S^{\prime \prime \prime}\left(x_{0}\right)+\frac{1}{2} S\left(x_{0}\right) S^{\prime \prime}\left(x_{0}\right)\right] } \\
& -\left[f^{\prime \prime \prime}\left(x_{0}\right)+\frac{1}{2} f\left(x_{0}\right) f^{\prime \prime}\left(x_{0}\right)\right] .
\end{aligned}
$$

Since $f(0)=S(0)=0$, we have the following result:

$$
\begin{aligned}
e(0) & =S^{\prime \prime \prime}(0)-f^{\prime \prime \prime}(0) \\
& =\left[-\frac{f^{(5)}(0)}{12}\right] h^{2}+\left[\frac{f^{(7)}(0)}{240}\right] h^{4}+O\left(h^{6}\right) .
\end{aligned}
$$

\section{Numerical Results}

In this section, approximation values of $f(x), f^{\prime}(x)$, and $f^{\prime \prime}(x)$ based on proposed methods for some values of $x$ 
TABLE 2: Comparison of $f(x)$ between some numerical results and our results using different values of $h$.

\begin{tabular}{|c|c|c|c|c|c|c|}
\hline$x$ & DTM [11] & LTNHPM [7] & Howarth (RK4) & $h=0.2$ & $\begin{array}{c}\text { posed me } \\
h=0.1\end{array}$ & $h=0.01$ \\
\hline 0.0 & 0.00000 & 0.00000 & 0.00000 & 0.00000 & 0.00000 & 0.00000 \\
\hline 0.2 & 0.00664 & 0.00664 & 0.00664 & 0.00664 & 0.00664 & 0.00664 \\
\hline 0.4 & 0.02656 & 0.02656 & 0.02656 & 0.02656 & 0.02656 & 0.02656 \\
\hline 0.6 & 0.05973 & 0.05973 & 0.05974 & 0.05973 & 0.05973 & 0.05973 \\
\hline 0.8 & 0.10611 & 0.10611 & 0.10611 & 0.10609 & 0.10610 & 0.10611 \\
\hline 1.0 & 0.16557 & 0.16557 & 0.16557 & 0.16554 & 0.16556 & 0.16557 \\
\hline 1.2 & 0.23795 & 0.23795 & 0.23795 & 0.23790 & 0.23794 & 0.23795 \\
\hline 1.4 & 0.32298 & 0.32298 & 0.32298 & 0.32290 & 0.32296 & 0.32298 \\
\hline 1.6 & 0.42032 & 0.42032 & 0.42032 & 0.42021 & 0.42029 & 0.42032 \\
\hline 1.8 & 0.52952 & 0.52952 & 0.52952 & 0.52936 & 0.52948 & 0.52952 \\
\hline 2.0 & 0.65002 & 0.65002 & 0.65003 & 0.64982 & 0.64997 & 0.65002 \\
\hline 2.2 & 0.78119 & 0.78119 & 0.78120 & 0.78093 & 0.78113 & 0.78119 \\
\hline 2.4 & 0.92229 & 0.92228 & 0.92230 & 0.92197 & 0.92221 & 0.92229 \\
\hline 2.6 & 1.07250 & 1.07250 & 1.07252 & 1.07212 & 1.07241 & 1.07250 \\
\hline 2.8 & 1.23098 & 1.23098 & 1.23099 & 1.23054 & 1.23087 & 1.23098 \\
\hline 3.0 & 1.39681 & 1.39681 & 1.39682 & 1.39631 & 1.39668 & 1.39681 \\
\hline 3.2 & 1.56909 & 1.56909 & 1.56911 & 1.56854 & 1.56896 & 1.56909 \\
\hline 3.4 & 1.74695 & 1.74695 & 1.74696 & 1.74635 & 1.74680 & 1.74695 \\
\hline 3.6 & 1.92952 & 1.92952 & 1.92954 & 1.92889 & 1.92936 & 1.92952 \\
\hline 3.8 & 2.11603 & 2.11602 & 2.11605 & 2.11536 & 2.11586 & 2.11603 \\
\hline 4.0 & 2.30574 & 2.30575 & 2.30576 & 2.30506 & 2.30557 & 2.30574 \\
\hline 4.2 & 2.49804 & 2.49805 & 2.49806 & 2.49733 & 2.49786 & 2.49804 \\
\hline 4.4 & 2.69236 & 2.69242 & 2.69238 & 2.69164 & 2.69218 & 2.69236 \\
\hline 4.6 & 2.88824 & 2.88859 & 2.88826 & 2.88751 & 2.88806 & 2.88824 \\
\hline 4.8 & 3.08532 & 3.08718 & 3.08534 & 3.08458 & 3.08513 & 3.08532 \\
\hline 5.0 & 3.28327 & 3.29272 & 3.28329 & 3.28252 & 3.28308 & 3.28327 \\
\hline
\end{tabular}

TABLE 3: Comparison of $f^{\prime}(x)$ between some numerical results and our results using different values of $h$.

\begin{tabular}{|c|c|c|c|c|c|c|}
\hline$x$ & DTM [11] & LTNHPM [7] & Howarth (RK4) & $h=0.2$ & $\begin{array}{c}\text { posed met } \\
h=0.1\end{array}$ & $h=0.01$ \\
\hline 0.0 & 0.00000 & 0.00000 & 0.00000 & 0.00000 & 0.00000 & 0.00000 \\
\hline 0.2 & 0.06641 & 0.06641 & 0.06641 & 0.06640 & 0.06641 & 0.06641 \\
\hline 0.4 & 0.13276 & 0.13276 & 0.13277 & 0.13275 & 0.13276 & 0.13276 \\
\hline 0.6 & 0.19894 & 0.19894 & 0.19894 & 0.19890 & 0.19893 & 0.19894 \\
\hline 0.8 & 0.26471 & 0.26471 & 0.26471 & 0.26465 & 0.26469 & 0.26471 \\
\hline 1.0 & 0.32978 & 0.32978 & 0.32979 & 0.32969 & 0.32976 & 0.32978 \\
\hline 1.2 & 0.39378 & 0.39378 & 0.39378 & 0.39365 & 0.39374 & 0.39378 \\
\hline 1.4 & 0.45626 & 0.45626 & 0.45627 & 0.45610 & 0.45622 & 0.45626 \\
\hline 1.6 & 0.51676 & 0.51676 & 0.51676 & 0.51656 & 0.51671 & 0.51676 \\
\hline 1.8 & 0.57476 & 0.57476 & 0.57477 & 0.57452 & 0.57470 & 0.57476 \\
\hline 2.0 & 0.62976 & 0.62977 & 0.62977 & 0.62950 & 0.62970 & 0.62976 \\
\hline 2.2 & 0.68131 & 0.68131 & 0.68132 & 0.68102 & 0.68124 & 0.68131 \\
\hline 2.4 & 0.72898 & 0.72898 & 0.72899 & 0.72868 & 0.72891 & 0.72898 \\
\hline 2.6 & 0.77245 & 0.77245 & 0.77246 & 0.77215 & 0.77238 & 0.77245 \\
\hline 2.8 & 0.81151 & 0.81151 & 0.81152 & 0.81122 & 0.81144 & 0.81151 \\
\hline 3.0 & 0.84604 & 0.84604 & 0.84605 & 0.84577 & 0.84598 & 0.84604 \\
\hline 3.2 & 0.87608 & 0.87608 & 0.87609 & 0.87584 & 0.87602 & 0.87608 \\
\hline 3.4 & 0.90176 & 0.90176 & 0.90177 & 0.90155 & 0.90171 & 0.90176 \\
\hline 3.6 & 0.92333 & 0.92333 & 0.92333 & 0.92315 & 0.92329 & 0.92333 \\
\hline 3.8 & 0.94112 & 0.94112 & 0.94112 & 0.94098 & 0.94108 & 0.94112 \\
\hline 4.0 & 0.95552 & 0.95553 & 0.95552 & 0.95541 & 0.95549 & 0.95552 \\
\hline 4.2 & 0.96696 & 0.96704 & 0.96696 & 0.96688 & 0.96694 & 0.96696 \\
\hline 4.4 & 0.97587 & 0.97639 & 0.97587 & 0.97581 & 0.97586 & 0.97587 \\
\hline 4.6 & 0.98268 & 0.98564 & 0.98269 & 0.98264 & 0.98267 & 0.98268 \\
\hline 4.8 & 0.98779 & 1.00322 & 0.98779 & 0.98775 & 0.98778 & 0.98779 \\
\hline 5.0 & 0.99154 & 1.06671 & 0.99155 & 0.99151 & 0.99153 & 0.99154 \\
\hline
\end{tabular}


TABLE 4: Comparison of $f^{\prime \prime}(x)$ between some numerical results and our results using different values of $h$.

\begin{tabular}{|c|c|c|c|c|c|c|}
\hline$x$ & DTM [11] & LTNHPM [7] & Howarth (RK4) & $h=0.2$ & $\begin{array}{c}\text { oosed me } \\
h=0.1\end{array}$ & $h=0.01$ \\
\hline 0.0 & 0.33206 & 0.33206 & 0.33206 & 0.33206 & 0.33206 & 0.33206 \\
\hline 0.2 & 0.33198 & 0.33198 & 0.33199 & 0.33195 & 0.33197 & 0.33198 \\
\hline 0.4 & 0.33147 & 0.33147 & 0.33147 & 0.33140 & 0.33145 & 0.33147 \\
\hline 0.6 & 0.33008 & 0.33008 & 0.33008 & 0.32997 & 0.33005 & 0.33008 \\
\hline 0.8 & 0.32739 & 0.32739 & 0.32739 & 0.32725 & 0.32735 & 0.32739 \\
\hline 1.0 & 0.32301 & 0.32301 & 0.32301 & 0.32284 & 0.32297 & 0.32301 \\
\hline 1.2 & 0.31659 & 0.31659 & 0.31659 & 0.31641 & 0.31654 & 0.31659 \\
\hline 1.4 & 0.30786 & 0.30786 & 0.30787 & 0.30767 & 0.30782 & 0.30786 \\
\hline 1.6 & 0.29666 & 0.29666 & 0.29667 & 0.29648 & 0.29662 & 0.29666 \\
\hline 1.8 & 0.28293 & 0.28293 & 0.28293 & 0.28276 & 0.28289 & 0.28293 \\
\hline 2.0 & 0.26675 & 0.26675 & 0.26675 & 0.26662 & 0.26672 & 0.26675 \\
\hline 2.2 & 0.24835 & 0.24835 & 0.24835 & 0.24826 & 0.24833 & 0.24835 \\
\hline 2.4 & 0.22809 & 0.22809 & 0.22809 & 0.22805 & 0.22808 & 0.22809 \\
\hline 2.6 & 0.20645 & 0.20645 & 0.20646 & 0.20647 & 0.20646 & 0.20645 \\
\hline 2.8 & 0.18401 & 0.18401 & 0.18401 & 0.18408 & 0.18402 & 0.18401 \\
\hline 3.0 & 0.16136 & 0.16136 & 0.16136 & 0.16148 & 0.16139 & 0.16136 \\
\hline 3.2 & 0.13913 & 0.13913 & 0.13913 & 0.13928 & 0.13917 & 0.13913 \\
\hline 3.4 & 0.11788 & 0.11788 & 0.11788 & 0.11805 & 0.11792 & 0.11788 \\
\hline 3.6 & 0.09809 & 0.09809 & 0.09809 & 0.09827 & 0.09813 & 0.09809 \\
\hline 3.8 & 0.08013 & 0.08014 & 0.08013 & 0.08030 & 0.08017 & 0.08013 \\
\hline 4.0 & 0.06423 & 0.06436 & 0.06424 & 0.06438 & 0.06427 & 0.06423 \\
\hline 4.2 & 0.05052 & 0.05131 & 0.05052 & 0.05064 & 0.05055 & 0.05052 \\
\hline 4.4 & 0.03897 & 0.04360 & 0.03897 & 0.03906 & 0.03899 & 0.03897 \\
\hline 4.6 & 0.02948 & 0.05446 & 0.02948 & 0.02954 & 0.02950 & 0.02948 \\
\hline 4.8 & 0.02187 & 0.14672 & 0.02187 & 0.02190 & 0.02188 & 0.02187 \\
\hline 5.0 & 0.01591 & 0.59821 & 0.01591 & 0.01591 & 0.01591 & 0.01591 \\
\hline
\end{tabular}

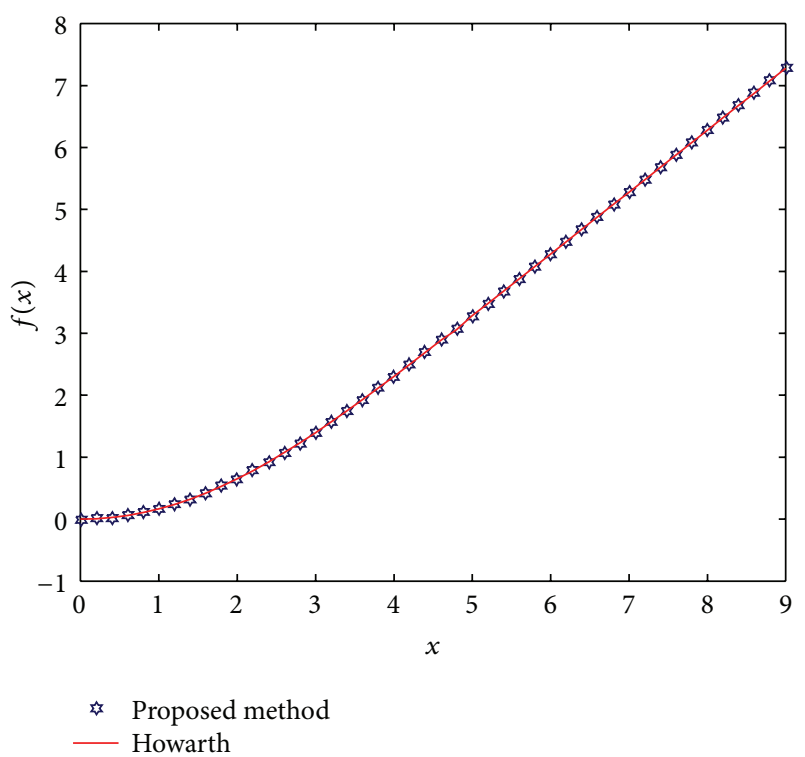

Figure 1: The comparison of $f(x)$ between our results and Howarth's results with $h=0.01$.

are presented. These values for $h=0.2,0.1,0.01$ have been calculated and these results are compared with the Howarth results. Tables 2, 3, and 4 are made to compare between the present results and results given by Howarth for approximation values of $f(x), f^{\prime}(x)$, and $f^{\prime \prime}(x)$, respectively. In Figures 1, 2, and 3, one can also see the comparison between our results and Howarth's results.

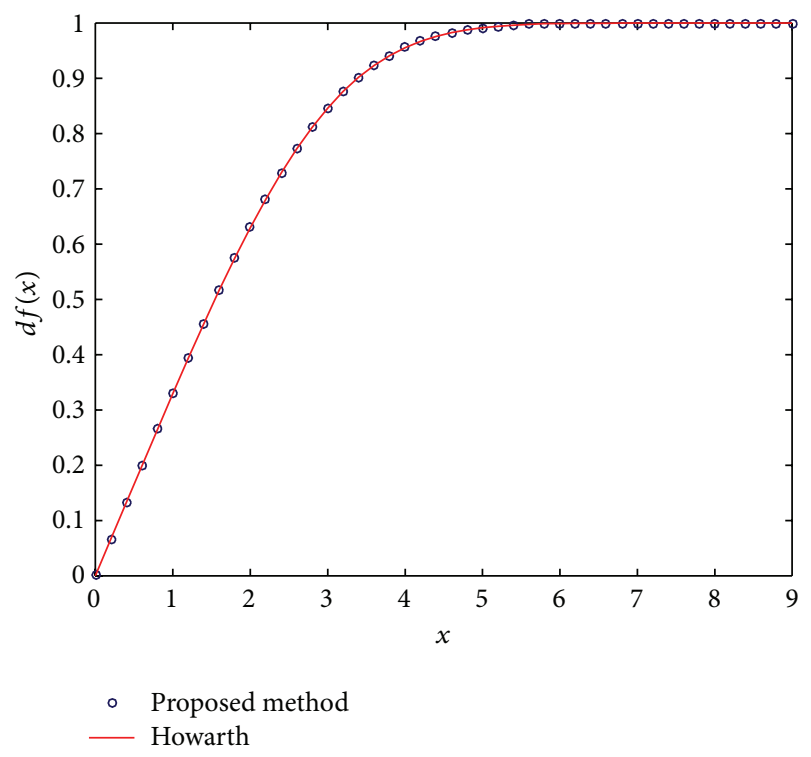

FIGURE 2: The comparison of $f^{\prime}(x)$ between our results and Howarth's results with $h=0.01$.

\section{Conclusion}

In this survey, the quartic B-spline approximations are used to solve the Blasius equation. This method led to a system of nonlinear equations. The unknowns are obtained by minimizing the error norm. The computed results are compared with those of DTM, LTNHPM, and Howarth's methods to 


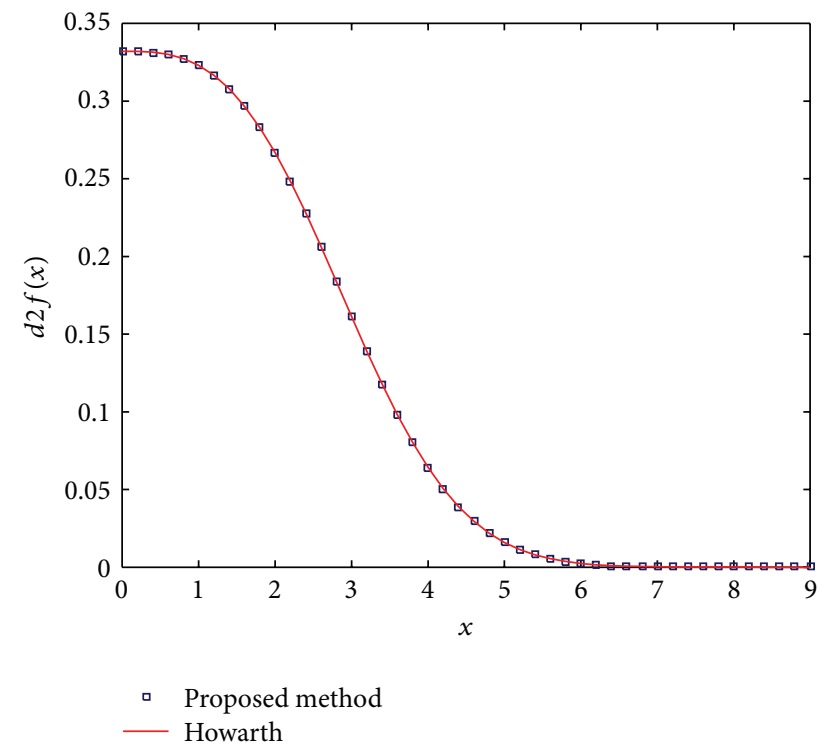

FIGURE 3: The comparison of $f^{\prime \prime}(x)$ between our results and Howarth's results with $h=0.01$.

demonstrate the validity and applicability of the technique. This method is simple in applicability and the results show that the solutions will become more accurate with reducing step size. The computations associated with the examples in this paper were performed using MATLAB R2015a.

\section{Competing Interests}

The authors of the paper do not have a direct financial relation that might lead to "competing interests" for any of the authors.

\section{References}

[1] H. Blasius, "Grenzschichten in flüssigkeiten mit kleiner reibung," Zeitschrift für Angewandte Mathematik und Physik, vol. 56, pp. 1-37, 1908.

[2] L. Howarth, "On the solution of the laminar boundary layer equations," Proceedings of the Royal Society A: Mathematical, Physical and Engineering Sciences, vol. 164, no. 919, pp. 547-579, 1938.

[3] C.-S. Liu and J.-R. Chang, "The Lie-group shooting method for multiple-solutions of Falkner-Skan equation under suctioninjection conditions," International Journal of Non-Linear Mechanics, vol. 43, no. 9, pp. 844-851, 2008.

[4] J. H. He, "Approximate analytical solution of Blasius' equation," Communications in Nonlinear Science and Numerical Simulation, vol. 3, no. 4, pp. 260-263, 1998.

[5] J.-H. He, "A simple perturbation approach to Blasius equation," Applied Mathematics and Computation, vol. 140, no. 2-3, pp. 217-222, 2003.

[6] C. M. Bender, K. A. Milton, S. S. Pinsky, and J. L. M. Simmons, "A new perturbative approach to nonlinear problems," Journal of Mathematical Physics, vol. 30, no. 7, pp. 1447-1455, 1989.

[7] H. Aminikhah, "Analytical approximation to the solution of nonlinear Blasius' viscous flow equation by LTNHPM," ISRN
Mathematical Analysis, vol. 2012, Article ID 957473, 10 pages, 2012.

[8] D. Xu and X. Guo, "Fixed point analytical method for nonlinear differential equations," Journal of Computational and Nonlinear Dynamics, vol. 8, no. 1, Article ID 011005, 2013.

[9] L. Wang, "A new algorithm for solving classical Blasius equation," Applied Mathematics and Computation, vol. 157, no. 1, pp. 1-9, 2004.

[10] R. Fazio, "Blasius problem and Falkner-Skan model: Topfer's algoritm and its extension," Computers \& Fluids, vol. 73, pp. 202-209, 2013.

[11] S. A. Lal and N. M. Paul, "An accurate taylors series solution with high radius of convergence for the Blasius function and parameters of asymptotic variation," Journal of Applied Fluid Mechanics, vol. 7, no. 4, pp. 557-564, 2014.

[12] C. Deboor, A Practical Guide to Splines, Springer, Berlin, Germany, 1978.

[13] P. M. Prenter, Splines and Variational Methods, John Wiley \& Sons, New York, NY, USA, 1989.

[14] J. Goh, A. A. Majid, and A. I. M. Ismail, "A quartic B-spline for second-order singular boundary value problems," Computers and Mathematics with Applications, vol. 64, no. 2, pp. 115-120, 2012. 


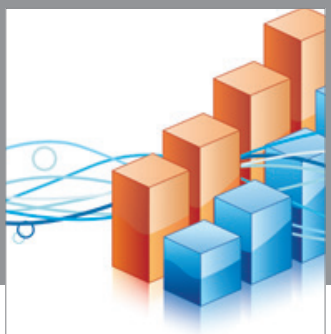

Advances in

Operations Research

vatem alat4

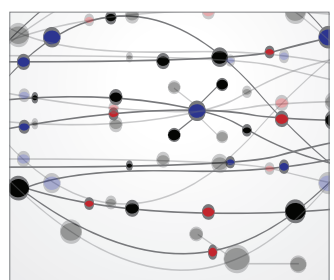

\section{The Scientific} World Journal
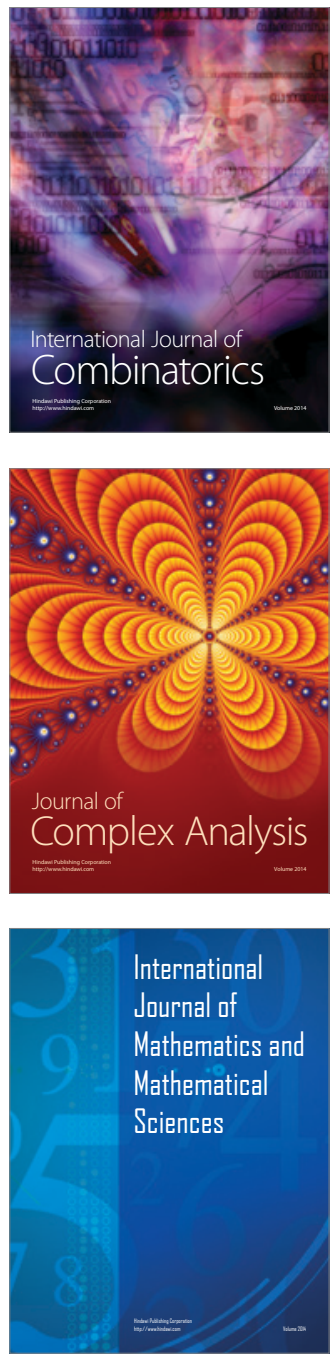
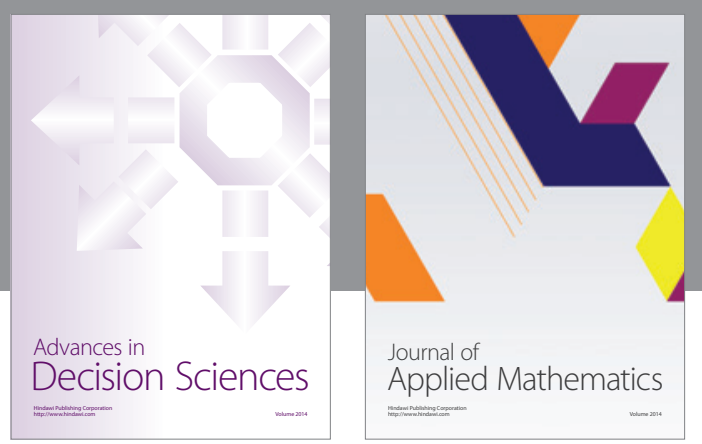

Algebra

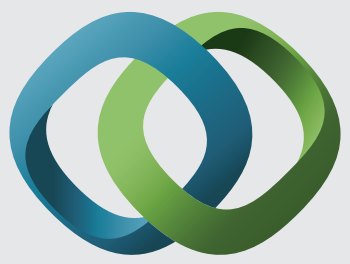

\section{Hindawi}

Submit your manuscripts at

http://www.hindawi.com
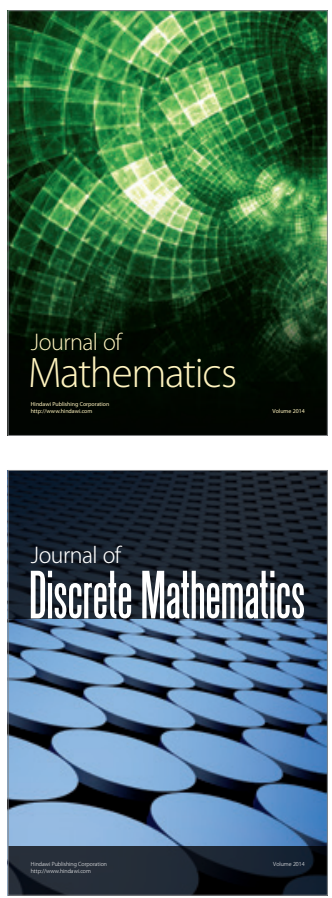

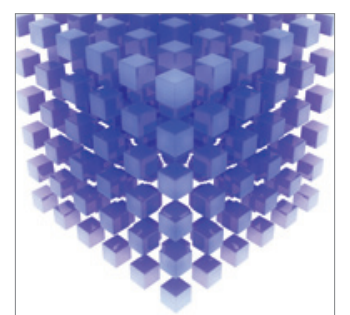

Mathematical Problems in Engineering
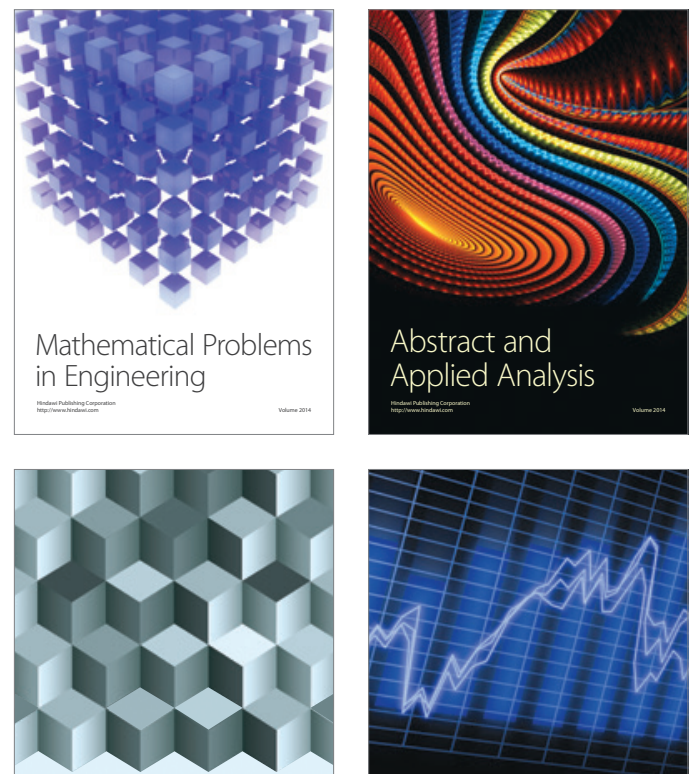

Journal of

Function Spaces

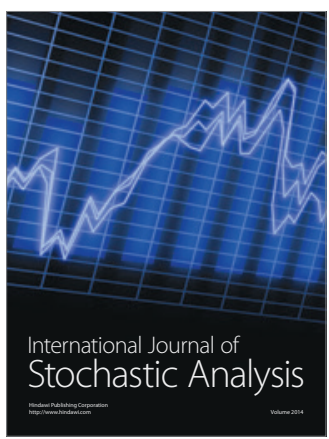

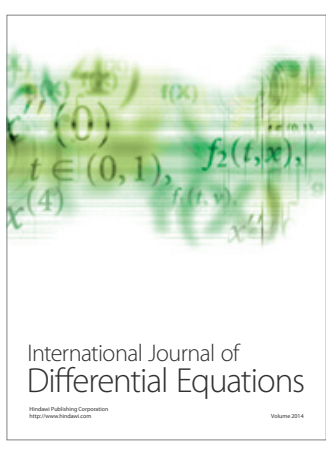
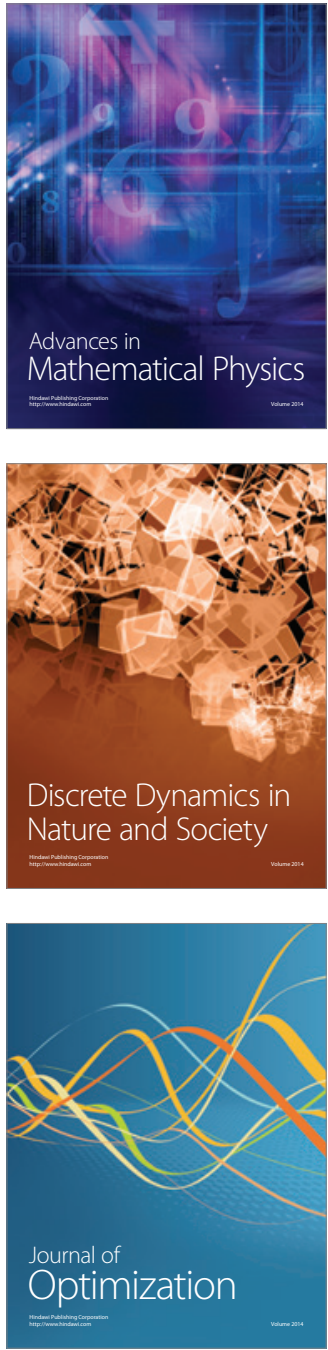